concentration fell significantly in all three patients, two of the patients showed a reduction in sperm motility, and the other patient showed a fall in the percentage of morphologically normal sperm. Despite the small number of patients included in the study, a statistically significant difference was found between the BCG and mitomycin $\mathrm{C}$ groups in terms of post-treatment sperm concentration $(P=0.0369)$.

In summary, these findings suggest that sperm quality may deteriorate following intravesical treatment with BCG. While larger studies are needed to confirm this, the authors recommend that routine pretreatment semen preservation should be offered to young men undergoing this therapy.

Original article Raviv G et al. (2005) Effects of intravesical chemotherapy and immunotherapy on semen analysis. Urology 65: 765-767

\section{Penile modeling for erectile dysfunction in Peyronie's disease}

A recent retrospective study has revealed that the combinination of penile modeling with the established procedure of insertion of an inflatable penile prosthesis, results in high levels of patient satisfaction and correction of erectile dysfunction in men with Peyronie's disease.

Chaudhary et al. carried out a postoperative evaluation of 46 patients who had undergone insertion of a prosthesis, 28 of whom had subsequently undergone a penile modeling procedure, which involved forcibly bending the penis in a direction opposite to that of the curvature. Patients were evaluated both in the clinic and by postal questionnaire up to 5 years following the procedure, and were questioned about their erectile function and satisfaction levels with the prosthesis.

The vast majority of patients (96\%) had an intact penile prosthesis at follow up, and of these, $93 \%$ reported satisfaction with the prosthesis and penile modeling where this had been carried out, in addition to a significant improvement in erectile function. None of the patients required further surgery to improve the straightening of the penis.

The authors conclude that high satisfaction rates can be achieved for the correction of penile curvature in cases of Peyronie's disease-associated erectile dysfunction by combining insertion of an inflatable penile implant with the use of penile modeling. Patients should therefore be offered this modeling procedure as an additional treatment option.

Original article Chaudhary M et al. (2005) Peyronie's disease with erectile dysfunction: penile modeling over inflatable penile prostheses. Urology 65: 760-764

\section{Transperineal saturation prostate biopsy}

The detection of prostate cancer among highrisk individuals remains a challenge because false-negative rates are high using standard biopsy techniques. Pinkstaff and colleagues from the Mayo Clinic in Jacksonville, Florida, have recently reported on their three-year experience of an alternative approach: systematic transperineal ultrasound-guided template biopsy.

The investigators prospectively recruited 210 men of mean age 66.3 years, with a mean serum PSA level of $13.6 \pm 10.2 \mathrm{ng} / \mathrm{ml}$. All had previously had at least one set of negative transrectal prostate biopsies; the mean number of negative cores was 17.4. Transperineal template prostate biopsy was performed on all patients, with the number of cores ranging from 12 to 41 (mean 21.2).

Despite the negative results of previous biopsies, systematic transperineal template biopsy led to a diagnosis of adenocarcinoma of the prostate in 78 (37\%) of these high-risk patients. The majority of cases $(77 \%)$ involved the detection of cancer in the transition zone, and there were 36 (46\%) transition-zone-only tumors. Positive results were significantly associated with increasing age and with a smaller prostate volume, but not with PSA level, the number of previous negative cores, or the number of template biopsy cores.

Commenting that the technique is easy to perform and uses standard brachytherapy equipment, Pinkstaff et al. conclude that systematic transperineal template biopsy of the prostate improves the rate of detection of transition-zone cancers in this setting.

\footnotetext{
Original article Pinkstaff DM et al. (2005) Systematic transperineal ultrasound-guided template biopsy of the prostate: three-year experience. Urology 65: 735-739
} 